\title{
Cognitive Enhancement via Neuromodulation and Video Games: Synergistic Effects?
}

\author{
Marc Palaus, Raquel Viejo-Sobera*, Diego Redolar-Ripoll ${ }^{\dagger}$ and Elena M. Marrón ${ }^{\dagger}$ \\ Cognitive NeuroLab, Faculty of Health Sciences, Universitat Oberta de Catalunya (UOC), Barcelona, Spain
}

Transcranial magnetic stimulation (TMS) is a non-invasive brain stimulation technique able to modulate cortical excitability. This modulation may influence areas and networks

OPEN ACCESS

Edited by:

Paul Sauseng,

Ludwig Maximilian University of Munich, Germany

Reviewed by:

Patrick Müller,

German Center for

Neurodegenerative Diseases (DZNE),

Germany

Elvire Vaucher,

Université de Montréal, Canada

*Correspondence:

Raquel Viejo-Sobera

rviejos@uoc.edu

tThese authors share senior authorship

Specialty section:

This article was submitted to Cognitive Neuroscience, a section of the journal Frontiers in Human

Neuroscience

Received: 02 March 2020 Accepted: 28 May 2020

Published: 19 June 2020

Citation:

Palaus M, Viejo-Sobera $R$, Redolar-Ripoll $D$ and Marrón EM (2020) Cognitive Enhancement via Neuromodulation and Video Games:

Synergistic Effects?

Front. Hum. Neurosci. 14:235. doi: 10.3389/fnhum.2020.00235 responsible for specific cognitive processes, and the repetition of the induced temporary changes can produce long-lasting effects. TMS effectiveness may be enhanced when used in conjunction with cognitive training focused on specific cognitive functions. Playing video games can be an optimal cognitive training since it involves different cognitive components and high levels of engagement and motivation. The goal of this study is to assess the synergistic effects of TMS and video game training to enhance cognition, specifically, working memory and executive functions. We conducted a randomized $2 \times 3$ repeated measures (stimulation $\times$ time) study, randomly assigning 27 healthy volunteers to an active intermittent theta-burst stimulation or a sham stimulation group. Participants were assessed using a comprehensive neuropsychological battery before, immediately after, and 15 days after finishing the video game+TMS training. The training consisted of 10 sessions where participants played a 3D platform video game for $1.5 \mathrm{~h}$. After each gaming session, TMS was applied over the right dorsolateral prefrontal cortex (DLPFC). All participants improved their video gaming performance, but we did not find a synergistic effect of stimulation and video game training. Neither had we found cognitive improvements related to the stimulation. We explored possible confounding variables such as age, gender, and early video gaming experience through linear regression. The early video gaming experience was related to improvements in working memory and inhibitory control. This result, although exploratory, highlights the influence of individual variables and previous experiences on brain plasticity.

Keywords: cognitive enhancement, dorsolateral prefrontal cortex, executive functions, iTBS, theta-burst stimulation, transcranial magnetic stimulation, video games, working memory

Abbreviations: BF, Bayes Factor; DLPFC, dorsolateral prefrontal cortex; Exp, participants with early video game experience; GLM, general linear model; iTBS, intermittent theta-burst stimulation; MRI, magnetic resonance imaging; NoExp, participants without early video game experience; rm GLM, repeated measures GLM; SSRT, stop-signal reaction time; TMS, transcranial magnetic stimulation; TMS+VG, TMS together with video game training. 


\section{INTRODUCTION}

Non-invasive brain stimulation techniques have become a step forward in cognitive neuroscience due to their ability to establish causal links between cognition and its neural substrate. Among these techniques, transcranial magnetic stimulation (TMS) allows modulation of cortical excitability in highly specific target regions, inducing changes in the associated cognitive functions and even enhancing them (e.g., Luber and Lisanby, 2014).

Nevertheless, the specific parameters through which TMS affects cognition are not entirely clear. TMS effectiveness seems to be partially task-dependent (Koch and Rothwell, 2009; Johnson et al., 2012; Duecker et al., 2013; Matsugi et al., 2014) and most effective when used together with cognitive training (Bentwich et al., 2011; Schilberg et al., 2012; Hopfner et al., 2015; Rabey and Dobronevsky, 2016; Lee et al., 2017; Nguyen et al., 2017). But both, the stimulation and the training must have certain characteristics to achieve the desired near-transfer and far-transfer effects (i.e., translation of benefits to similar or different cognitive domains, respectively).

Regarding the stimulation, its influence can be maximized when it is delivered after skill training. This allows us to take advantage of the state-dependency effects in specific neural populations (Romei et al., 2016), and TMS can interact with their current, imbalanced state (Silvanto et al., 2018). Examples in animal studies, using hypothalamic intracranial self-stimulation have shown that, administering the stimulation immediately after a skill training produce higher retention rates for that skill than administering the stimulation before the training (Redolar-Ripoll et al., 2002).

On the other hand, transfer effects of the training are maximized when different cognitive skills are integrated (Taatgen, 2013), high levels of engagement and motivation are maintained (Maraver et al., 2016), and there is sufficient exposure to the task (Zhao et al., 2020). In recent decades, video games have received a great deal of attention as cognitive training tools, due to some features that make them suitable for cognitive enhancement: they are widely available, integrate several cognitive processes at once, allow adjustment of variable difficulty, and are highly motivating and engaging. Furthermore, they are often used for long enough over a person's lifetime to have a real impact on cognition. There is a considerable body of literature dedicated to the effects of video gaming on the brain (for a systematic review see Palaus et al., 2017), and the implications of using a particular video game genres are well understood (Dobrowolski et al., 2015).

Given the ability of TMS to induce plastic changes in the brain, and the particular suitability of video games to train cognitive functions, we expect that their combination would produce synergistic effects on cognitive enhancement, but the literature documenting combined use of TMS and video games is still scarce (e.g., Anguera et al., 2013). In particular, we expect to enhance cognition (i.e., processing speed, visuospatial skills, attention, working memory, executive functions, and general intelligence) in a healthy sample by playing a $3 \mathrm{D}$ platform video game during 10 sessions and applying TMS over the dorsolateral prefrontal cortex (DLPFC) immediately after playing. We hypothesized that post video game TMS would enhance the positive effects of video game training over cognition.

\section{MATERIALS AND METHODS}

\section{Participants}

While 32 participants were recruited, five did not complete all the phases of the study (two due to discomfort during the stimulation, two due to incompatibility of schedules, and 1 for undisclosed personal reasons). The final sample was therefore composed of 27 healthy adults ( 14 women and 13 men) aged $18-40$ years $(29.44 \pm 6.28)$. Participants were excluded if they had neurological or psychiatric disorders, including depression, measured through Beck's Depression Inventory (BDI-II, Beck et al., 1996), were abusers of drugs or alcohol, did not meet safety criteria for both magnetic resonance imaging (MRI) and TMS (Rossi et al., 2009), and played video games (of any kind) for more than $3 \mathrm{~h}$ /week at the time of the study. Those who had previously played the video game used in this study (Super Mario 64 ) or any of its sequels, regardless of the experience level, were also excluded.

The study was approved by the Universitat Oberta de Catalunya (UOC) Ethics Committee. All participants gave written informed consent to participate in the study following the Declaration of Helsinki and received monetary compensation amounting to 80 euros.

\section{Design}

A randomized $2 \times 3$ repeated measures (stimulation $\times$ time) study was conducted. Participants were randomly allocated to one of two stimulation groups: Active or Sham and evaluated before, immediately after the training period, and 15 days after finishing the training. When comparing the outcome measures of the two experimental groups, differences after training were only found in one of the variables (see "Results" section). Therefore, we decided to further explore the data to identify possible confounding variables that might have influenced the results.

Logistic regression was implemented to identify potential predictors of cognitive changes after the TMS+VG training period (see "Data Analysis" and "Results" section). Based on previous literature on video gaming and its effects on brain structure and function (see Palaus et al., 2017), we considered age, gender, and early video gaming experience as possible predictor variables. In line with previous studies (Hartanto et al., 2016; Palaus et al., 2017), early video game experience was defined as having played regularly before adolescence (14 years old or younger) for at least 1 year and more than $3 \mathrm{~h}$ /week (information collected through an ad hoc guided interview). The results of the logistic regression showed that early video gaming experience (in any kind of video game genre) predicted some of the performance changes observed in executive function tasks (see "Results" section). We, therefore, used this variable to classify the sample into four subgroups (see Table 1) according to stimulation modality (Active vs. Sham) and video game experience (Exp vs. NoExp). 
TABLE 1 | Characteristics of the groups (size, age \pm standard deviation, and gender).

\begin{tabular}{|c|c|c|c|}
\hline & Active & Sham & TOTAL \\
\hline Experienced gamer & $n=6$ (1 female) Age: $28.11 \pm 7.23$ & $n=6$ (2 female) Age: $28.33 \pm 7.32$ & $n=12$ (3 female) Age: $28.17 \pm 7.30$ \\
\hline Non-experienced gamer & $n=8$ (6 female) Age: $32.50 \pm 3.70$ & $n=7$ (5 female) Age: $29.72 \pm 7.00$ & $n=15$ (11 female) Age: $30.47 \pm 5.38$ \\
\hline TOTAL & $n=14$ (7 female) Age: $29.86 \pm 5.26$ & $n=13$ (7 female) Age: $29.00 \pm 7.43$ & $n=27$ (14 female) Age: $29.44 \pm 6.28$ \\
\hline
\end{tabular}

\section{Procedure}

The participants were enrolled in the study for 1 month, during which they participated in 10 TMS+VG sessions. They were assessed at three time points: before training started (Pre) after training ended (Post1), and 15 days after the end of the training (Post2; see Figure 1 and Table 2). Assessments and training procedures are described in what follows.

\section{Neuropsychological Assessment}

The participants were assessed using eight tasks (as listed in Table 2 and described in Table 3) to obtain a comprehensive measure of their cognitive abilities, with a special focus on executive functions and working memory. Not all tasks were administered at each assessment point due to the high risk of practice effects associated with some of them (e.g., the matchstick task; see Table 2). Except for the five-point test, all tasks were programmed using the E-Prime 2.0 software and computeradministered (for a full description see Palaus, 2018).

\section{Video Game Training}

For the video game training we used Super Mario 64, a video game created by Nintendo in 1996. Super Mario 64 is a $3 \mathrm{D}$ platform game emphasizing exploration and puzzlesolving, requiring planning ability and goal-oriented behaviors. This video game was chosen because its use has shown a correlation with structural changes in the brain, i.e., an increase in cortical thickness of the right DLPFC, right hippocampal formation, and bilateral cerebellum, regions associated with executive functioning, spatial memory, and fine motor skills (Kühn et al., 2013).

Before and after the training, we assessed each participant's video gaming skills by getting them to play an alternative version of the same game used in the training sessions for $15 \mathrm{~min}$. We used the same level in both assessments. In particular, we assessed visuo-manual coordination, goal-oriented behavior, exploratory behavior, and behavior on facing obstacles and goal achievement (e.g., defeating an enemy, finding a hidden object, reaching a section of a level, etc.). This assessment was performed using a scale created ad hoc.

During training, participants played for $1.5 \mathrm{~h}$ at the rate of one session a day for 10 consecutive days excluding weekends (total $15 \mathrm{~h}$ ) under the supervision of a researcher. Game sessions were also video recorded for later analysis. The only instructions given to participants were on how to use the controller and on what the main goals of the game were at the beginning of the training; otherwise, they played as they wished. The number of goals per session, the number of tries per goal, and the time needed to achieve each goal were measured. Overall performance was calculated by dividing the total number of goals achieved during the entire training period by the total number of attempts (each attempt ended either when the participant "lost-a-life" or achieved a goal).

The gaming experience was measured subjectively at each video game training session using a Likert scale of 1-5. Participants scored motivation (their desire to play) before playing, and fun (enjoyment during gameplay) and frustration (dissatisfaction during gameplay) after playing and before TMS.

\section{Transcranial Magnetic Stimulation}

A total of 10 TMS sessions were conducted, one after each video game training session. In each TMS session, brief pre- and post-stimulation assessments were made, consisting of the Mini-Mental State Examination (MMSE; Lobo et al., 2002), well-being, and acute substance consumption (only prestimulation) to detect and prevent any possible adverse effects of the stimulation. The TMS procedures complied with the international safety guidelines (Rossi et al., 2009), and none of the participants wore eye makeup to avoid local pain in the orbital area (Redolar-Ripoll et al., 2015).

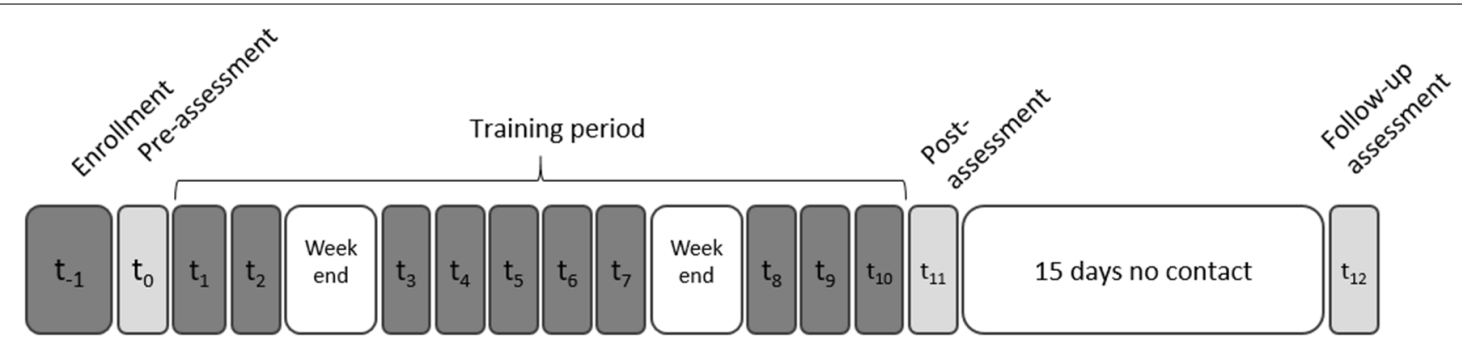

FIGURE 1 | Experimental timeline: neuropsychological assessments (light gray), video game training and transcranial magnetic stimulation (TMS) sessions (dark gray), and non-contact days (white). A structural magnetic resonance imaging (MRI) was obtained for each participant during the enrollment phase to: (1) discard the possibility of brain anomalies that could affect or prevent participation; and (2) locate the stimulation target and navigate the TMS coil position for each participant (see "Transcranial Magnetic Stimulation" section). 
TABLE 2 | Experimental schedule and phases following SPIRIT recommendations (Chan et al., 2013).

\begin{tabular}{|c|c|c|c|c|c|c|c|}
\hline \multirow[b]{3}{*}{ Time point } & \multicolumn{7}{|c|}{ Study period } \\
\hline & \multirow{2}{*}{$\begin{array}{c}\text { Enrollment } \\
\qquad t_{(-1)}\end{array}$} & \multirow{2}{*}{$\begin{array}{c}\text { Pre-assessment } \\
t_{(0)}\end{array}$} & \multicolumn{3}{|c|}{ TMS+VG training period } & \multicolumn{2}{|c|}{ Post-assessment } \\
\hline & & & $t_{(1)}$ & $t_{(2)}-t_{(9)}$ & $t_{(10)}$ & $t_{(11)}$ (post) & $t_{(12)}$ (15-day follow-up) \\
\hline \multicolumn{8}{|l|}{ Recruitment and allocation } \\
\hline Eligibility screening & $x$ & & & & & & \\
\hline Magnetic resonance imaging & $x$ & & & & & & \\
\hline Informed consent & & $x$ & & & & & \\
\hline Sociodemographic and video gaming data collection & & $x$ & & & & & \\
\hline Group allocation & & $x$ & & & & & \\
\hline \multicolumn{8}{|l|}{ Training } \\
\hline \multicolumn{8}{|l|}{ Video gaming (1.5 h) } \\
\hline \multicolumn{8}{|l|}{ Assessment } \\
\hline Active motor threshold & & & $x$ & & & & \\
\hline TMS screening & & & $x$ & $x$ & $\mathrm{x}$ & & \\
\hline Video game survey & & & $x$ & $x$ & $x$ & & \\
\hline Beck Depression Inventory & & $x$ & & & & $x$ & \\
\hline Mini-Mental State Examination & & & $x$ & $x$ & $x$ & & \\
\hline Reaction time tasks & & $x$ & & & & $x$ & $x$ \\
\hline Raven's progressive matrices & & $x$ & & & & $x$ & \\
\hline 3-Back task & & $x$ & & & & $x$ & $x$ \\
\hline Mental rotation task & & $x$ & & & & $x$ & $x$ \\
\hline Digit span tasks & & $x$ & & & & $x$ & $x$ \\
\hline Five-point test & & & & & & $x$ & \\
\hline Stop-switching task & & $x$ & & & & $x$ & $x$ \\
\hline Matchstick task & & & & & & $x$ & \\
\hline Video gaming skills & & $x$ & & & & $x$ & \\
\hline
\end{tabular}

TABLE 3 | Neuropsychological assessment tasks.

\begin{tabular}{|c|c|c|}
\hline Task (in the order administered) & Description and assessed cognitive functions & Collected data \\
\hline Reaction time tasks (Johnson et al., 1985) & $\begin{array}{l}\text { Three short visual reaction time tasks (simple, direction } \\
\text { choice and color choice), to assess processing speed }\end{array}$ & Accuracy and reaction times \\
\hline $\begin{array}{l}\text { Raven's progressive matrices (Raven, 1936; } \\
\text { Raven and Court, 2014) }\end{array}$ & $\begin{array}{l}\text { Two parallel versions (for the Pre and Post } 1 \text { assessments) of } \\
\text { standard Raven's progressive matrices, to measure general } \\
\text { intelligence }\end{array}$ & Accuracy and reaction times \\
\hline 3-back task (based on Salat et al., 2002) & $\begin{array}{l}\text { Visual continuous performance task, to measure working } \\
\text { memory }\end{array}$ & $\begin{array}{l}\text { Accuracy, reaction time, d' sensitivity } \\
\text { index [d' = Z(hit rate) }- \text { Z(false alarm } \\
\text { rate) as explained in Haatveit et al., } \\
2010]\end{array}$ \\
\hline $\begin{array}{l}\text { Mental rotation task (Shepard and Metzler, } \\
\text { 1971) }\end{array}$ & $\begin{array}{l}\text { Replicated Sheppard's mental rotation task using 3D } \\
\text { objects, to measure visuospatial skills }\end{array}$ & Accuracy and response time \\
\hline Digit span task (Wechsler, 2008) & $\begin{array}{l}\text { A computerized version of the WAIS-IV digit span test } \\
\text { presented acoustically, to measure attention span and } \\
\text { short-term memory (forward span) and working memory } \\
\text { (backward span) }\end{array}$ & Digit span (forward and backward) \\
\hline Stop-switching task (Obeso et al., 2013) & $\begin{array}{l}\text { Speed task combining go, stop, and switch trials, to } \\
\text { measure inhibition and task-switching components of } \\
\text { executive functions }\end{array}$ & $\begin{array}{l}\text { Accuracy and reaction time for go, stop } \\
\text { and switch trials, and stop-signal } \\
\text { reaction time (SSRT) }\end{array}$ \\
\hline Five-point test (Tucha et al., 2012) & $\begin{array}{l}\text { The paper-and-pencil task involving the connection of } \\
\text { patterns consisting of } 5 \text { dots under a time constraint, to } \\
\text { measure the ability to generate alternative solutions to a } \\
\text { problem }\end{array}$ & Accuracy \\
\hline Matchstick test (Knoblich et al., 1999) & $\begin{array}{l}\text { Math problem-solving task involving Roman numerals made } \\
\text { of sticks, as a measure of insight }\end{array}$ & Accuracy, reaction time \\
\hline
\end{tabular}

The stimulation target was set to Montreal Neurological Institute (MNI) coordinates $x=52, y=39, z=25$, corresponding to the point of maximum grey matter increase in the right DLPFC as a result of training in the video game (Kühn et al., 2013; see Figure 2). Coordinates were individually adjusted for each participant based on their structural MRI. For target location and
TMS guidance, we used the BrainSight 2 neuronavigation system (Rogue Research, Montreal, Canada).

Intermittent theta-burst stimulation (iTBS; Huang et al., 2005) was delivered following the international guidelines at $80 \%$ of the active motor threshold (Huang et al., 2005; Rossi et al., 2009; Suppa et al., 2016), using a Magstim Super 

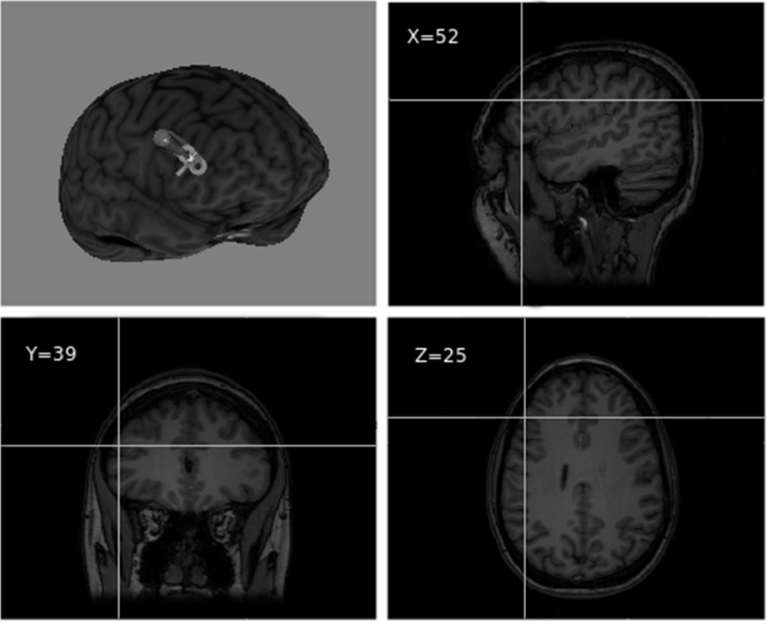

FIGURE 2 | Right dorsolateral prefrontal cortex (DLPFC) TMS target visualized over a 3D MRI reconstruction of a participant's head (reproduced with participant's permission).

Rapid 2 stimulator and a 70-mm figure-of-eight coil (Magstim Company Ltd., Whitland, UK). This stimulation protocol was chosen for its ability to induce long-term potentiation-like effects over the cerebral cortex (Goldsworthy et al., 2012) lasting up to 20 min (Suppa et al., 2016), with shorter stimulation times compared to other repetitive TMS protocols, but having similar effectiveness on clinical populations (e.g., Blumberger et al., 2018; Philip et al., 2019; Phillips et al., 2019). A total of 600 pulses were delivered in $50-\mathrm{Hz}$ triplets repeated at $5 \mathrm{~Hz}$ (i.e., every $200 \mathrm{~ms}$; Huang et al., 2011). This protocol involves repeating 2-s blocks of pulses interleaved with $8 \mathrm{~s}$ without stimulation 20 times during $200 \mathrm{~s}$.

The sham stimulation group received the same stimulation protocol, except that the coil was tilted $90^{\circ}$ over the vertex so that stimulation did not reach the cerebral cortex (Kaminski et al., 2011; Sandrini et al., 2011).

\section{Data Analysis}

We analyzed age differences between groups using Student's $t$ test (for comparisons of two groups) and one-way ANOVA (for comparisons of four subgroups). Gender differences between groups were calculated through Pearson's chi-square test.

Fitness to parametric assumptions was checked for all variables. To assess the normality of data distribution, the Shapiro-Wilk test was used. The homogeneity of variances was contrasted through the Levene's test of equality of variances. Lastly, to test sphericity, Mauchly's test was used when there were three levels of a repeated measure.

When parametric assumptions were not satisfied, task scores were transformed, and/or alternative non-parametric tests were employed, as explained below.

To compare video gaming and cognitive performance between Active and Sham groups, and taking into account the assessment time points for the tasks (2 or 3 depending on the task), a repeated measures general linear model ( $r m$ GLM) was implemented for each variable. Significant effects and interactions were followed-up using paired $t$-tests Bonferronicorrected for multiple comparisons. In measurements with just a single assessment point, the differences between groups were analyzed using the Student's $t$-test. To study differences between groups for variables that were not normally distributed, task scores were transformed calculating new scores reflecting prevs. post-training changes. Two difference scores between time points were calculated as follows: (1) pre-assessment vs. a first post-assessment difference (Pre-Post1); and (2) pre-assessment vs. follow-up assessment difference (Pre-Post2). ANOVAs were used to compare those difference scores between groups when parametric assumptions were satisfied, otherwise, we used the Mann-Whitney's U. When the distribution was normal, but equality of variances was not satisfied, we used Welch's ANOVA to compare scores between groups.

Additionally, given the small sample size that might limit the study power, we performed a set of Bayesian analyses to determine whether a non-significant effect indicates a lack of intervention effect (Biel and Friedrich, 2018). In particular, we tested the relative plausibility of the alternative hypothesis (H1: synergistic effects of Active TMS+video game training on cognitive performance) over the null hypothesis ( $\mathrm{H} 0$ : the absence of such synergistic effects, i.e., equal performance of Active and Sham TMS groups after training). Thus, we calculated BF10 using the Bayesian counterpart's tests of the analysis described above with a credible interval of $95 \%$. We used the default Cauchy prior width of 0.707 provided by JASP (JASP Team, 2020) since we did not have previous data to establish an informed prior. The models used for the analysis were compared to the model containing the grand mean and the random factors, called the null model. In the case of the independent sample $t$-test (Student or Mann-Whitney, see Supplementary Table S2) we use unidirectional hypothesis tests expecting cognitive improvement in the Active compared to the Sham TMS group.

As explained in the "Design" section, after performing the main comparison between the Active and Sham groups, and based on previous literature on video games showing that personal variables account for a sizeable portion of the variance (see Palaus et al., 2017), additional analyses were performed to observe the possible relationship between individual variables and cognitive changes. Logistic regression accounting for age, gender, and the early video gaming experience were performed for each dependent variable. The results indicated that early video gaming experience and gender were potentially linked to cognitive performance results, with video gaming experience more directly related to changes in executive functions (see "Results" section).

Therefore, an rm GLM was used to compare the variables significantly influenced by early video game experience for the four subgroups resulting after combining stimulation type and early video gaming experience (Active+Exp, $n=6$; Active+NoExp, $n=8$; Sham+Exp, $n=6$; and Sham+NoExp, $n=7)$. Parametric assumptions were also assessed for each variable for the four groups and we implemented the same 
procedures as above when assumptions were not met. But when, after transforming the scores, parametric assumptions were still not met, we used the Kruskal-Wallis $\mathrm{H}$ test to compare the four four subgroups, and when significant differences were found we used Mann-Whitney's U to compare the groups in pairs. Since performance for one of the variables was also predicted by gender (besides gaming experience), gender was included as a covariate in the corresponding analysis.

Finally, to control for the potential influence of the subjective gaming experience on video game performance and potential stimulation effects (Maraver et al., 2016), the Student's $t$-test was used to compare the Active and Sham groups for the motivation, fun, and frustration variables.

The dataset generated and analyzed during the current study, together with an explanatory readme file, is available in the institutional repository of the Universitat Oberta de Catalunya (O2), public URL: http://hdl.handle.net/10609/100246 (Palaus et al., 2019). All the frequentist analyses were performed using SPSS version 23 (IBM Software Group, IL, USA), and all the Bayesian analyses were performed in JASP computer software, version 0.12.2 (JASP Team, 2020).

\section{RESULTS}

\section{Demographic Data}

Age was not significantly different between the Active and Sham groups $\left(t_{(25)}=0.35, p=0.731\right)$ or between the four subgroups taking into account video gaming experience $\left(F_{(3,23)}=0.89\right.$; $p=0.457)$. There were no significant gender differences between the two initial groups (Active vs. Sham: $\chi_{(1)}^{2}=0.40 ; p=0.842$ ) or the four subgroups (Active+Exp, Active+NoExp, Sham+Exp, Sham+NoExp, $\left.\chi_{(1)}^{2}=6.59 ; p=0.086\right)$. Moreover, the number of participants with early video gaming experience was not significantly different between the Active and Sham groups $\left(\chi_{(1)}^{2}=0.03 ; p=0.863\right)$.

\section{Effects of the Transcranial Magnetic Stimulation}

\section{Frequentist Analyses}

When comparing video game performance (after the 10 sessions of training and the video game skills before and after training; i.e., near transfer) and cognitive test performance (i.e., far transfer) for the Active $(n=14)$ and Sham $(n=13)$ groups, most differences were not statistically significant. See Table 4 for further information on tests and significance levels for each contrast and see Supplementary Table S1 for descriptive statistics of each variable (mean, standard deviation, and confidence interval). Here we will only report the significant results and their post hoc analyses.

Some variables showed changes over time (main effect of time) that were similar for all groups, probably due to training and practice effects. Since our main interest was to compare the effects of TMS combined with video game training, that main effect of time was not specifically explored but was observed as part of the GLM analysis results and thus is only reported for variables that were normally distributed.
In particular, we observed improvement over time in video game skills $\left(F_{(1,25)}=101.95, p=0.000, \eta_{p}^{2}=0.80\right)$, digits forward (Pre vs. Post1 assessments $F_{(1,25)}=6.16, p=0.020$, $\left.\eta_{p}^{2}=0.20\right)$, 3-back task reaction times $\left(F_{(1.5,37.61)}=4.85\right.$ $\left.p=0.021, \eta_{p}^{2}=0.16\right)$, and accuracy $\left(F_{(2,50)}=5.88, p=0.005\right.$, $\left.\eta_{p}^{2}=0.19\right)$. In the Stop-switching task, Switch trials reaction times decreased $\left(F_{(2,50)}=3.82, p=0.029, \eta_{p}^{2}=0.13\right)$, but Go reaction times $\left(F_{(2,50)}=3.35, p=0.043, \eta_{p}^{2}=0.12\right)$ and stop signal reaction times (SSRT; Pre vs. Post1 $F_{(1,25)}=4.3$, $\left.p=0.049, \eta_{p}^{2}=0.15\right)$ increased. In the case of the simple reaction time task, results also deteriorated over time $\left(F_{(2,50)}=6.33\right.$; $\left.p=0.004, \eta_{p}^{2}=0.20\right)$. It should be noted, however, that this was also the only task showing significant interaction effects between the stimulation group and the assessment time points $\left(F_{(2,50)}=4.45 ; p=0.017, \eta_{p}^{2}=0.15\right.$; see Table 4). Post hoc analyses revealed that participants in the Active group had slower reaction times in the Post 2 assessment compared to both the Pre $(p=0.005$, mean difference $=28.3,95 \%$ CI 7.5-49.1 $)$ and the Post1 assessment ( $p=0.001$, mean difference $=25.6,95 \%$ CI 10.2-41.1). Interestingly enough, this effect was not observed for the more complex reaction time tasks (color and direction reaction time: $p>0.115$; see Table 4 ).

We also found statistically significant differences between the Active and Sham groups in some tasks (main effect of the group), given the absence of interaction effect between group and time, this was probably due to differences in baseline performance. This effect was observed for the 3-back d' score $\left(F_{(1,25)}=5.53, p=0.027, \eta_{p}^{2}=0.18\right)$ and for the time taken to complete Raven's progressive matrices $\left(F_{(1,25)}=7.41, p=0.012\right.$, $\left.\eta_{p}^{2}=0.23\right)$. In both cases, the Active group showed better scores than the Sham group and significant differences between groups at baseline $(p s<0.043)$.

\section{Bayesian Analyses}

The comparisons between the Active and Sham TMS stimulation groups using Bayesian methods yield very similar results than the ones obtained when performing the same comparisons through frequentist statistical analyses. However, the main effect of time only showed strong evidence in favor of the alternative hypothesis (i.e., BF greater than 10 following Jeffreys, 1961) for the improvement in video game skills $\left(\mathrm{BF}_{10}=6.575 \times 10^{7}\right.$, error\% $\left.=2.163\right)$. The rest of the variables showing changes in the previous analyses obtained weak (BF 1-3) to moderate (BF 3-10) evidence (see Supplementary Table S2 in the Supplementary Material). The only significant group by time interaction effect found through frequentist analysis for the simple reaction time task could not be replicated here $\left(\mathrm{BF}_{\text {incl }}=0.359\right.$; see Supplementary Table S2).

Regarding the few previously observed differences between groups, the evidence in favor of the alternative hypothesis was also weak to moderate. Overall, the Bayesian analysis reinforces the idea that the non-significant effects found via frequentist analyses support the null hypothesis.

\section{Logistic Regression of Individual Variables}

Age, gender, and early video gaming experience were explored through logistic regression analysis (using enter method), to 
TABLE 4 | Statistical tests, statistics, and significance levels for comparisons of video games and cognitive tests performance for the Active vs. Sham groups

\begin{tabular}{|c|c|c|c|c|c|c|c|}
\hline \multirow[b]{2}{*}{ Test } & \multirow[b]{2}{*}{ Variable } & \multirow[b]{2}{*}{$\begin{array}{c}\text { Assessment } \\
\text { time points }\end{array}$} & \multirow[b]{2}{*}{ Scores } & \multirow[b]{2}{*}{$\begin{array}{l}\text { Statistical } \\
\text { test }\end{array}$} & \multicolumn{2}{|c|}{ Main effects } & \multirow[b]{2}{*}{ Interaction } \\
\hline & & & & & Group & Time & \\
\hline \multirow{2}{*}{$\begin{array}{l}\text { Video game } \\
\text { performance }\end{array}$} & 10-session training & 1 & Direct & Mann-Whitney & $U=86.5, p=0.827$ & - & - \\
\hline & Pre vs. post skills & 2 & Direct & GLM & $F_{(1,25)}=0.121, p=0.731$ & $F_{(1,25)}=101.95, p<0.001$ & $F_{(1,25)}=0.757, p=0.393$ \\
\hline \multirow[t]{3}{*}{ RT } & Simple & 3 & Direct & GLM & $F_{(1,25)}=0.04 ; p=0.839$ & $F_{(2,50)}=6.33 ; p=0.004$ & $F_{(2,50)}=4.45 ; p=0.017$ \\
\hline & Direction choice & 3 & $\begin{array}{l}\text { Transformed (Post1-Pre, } \\
\text { Post2-Pre) }\end{array}$ & ANOVA & - & - & $\begin{array}{l}F_{(1,25)}=0.953, p=0.338 \\
F_{(1,25)}=2.663, p=0.115\end{array}$ \\
\hline & Color choice & 3 & $\begin{array}{l}\text { Transformed (Post1-Pre, } \\
\text { Post2-Pre) }\end{array}$ & ANOVA & - & - & $\begin{array}{l}F_{(1,25)}=1.459, p=0.238 \\
F_{(1,25)}=0.927, p=0.345\end{array}$ \\
\hline \multirow[t]{3}{*}{ Digits } & Forward & 3 & Direct (Pre vs. Post1) & GLM & $F_{(1,25)}=0.015, p=0.903$ & $F_{(1,25)}=6.156, p=0.020$ & $F_{(1,25)}=0.008, p=0.927$ \\
\hline & & & Transformed (Post2-Pre) & ANOVA & & - & $F_{(1,25)}=0.037, p=0.849$ \\
\hline & Backward & 3 & $\begin{array}{l}\text { Transformed (Post1-Pre, } \\
\text { Post2-Pre) }\end{array}$ & ANOVA & - & - & $\begin{array}{l}F_{(1,25)}=0.028, p=0.867 \\
F_{(1,25)}=0.048, p=0.828\end{array}$ \\
\hline \multirow[t]{3}{*}{ 3-back } & Score & 3 & $\begin{array}{l}\text { Transformed (Post1-Pre, } \\
\text { Post2-Pre) }\end{array}$ & ANOVA & - & - & $\begin{array}{l}F_{(1,25)}=0.851, p=0.365 \\
F_{(1,25)}=0.1 .085, p=0.308\end{array}$ \\
\hline & RT & 3 & $\begin{array}{l}\text { Direct (Greenhouse-Geisser } \\
\text { correction) }\end{array}$ & GLM & $F_{(1,25)}=0.123, p=0.729$ & $F_{(1.5,37.6)}=4.852 p=0.021$ & $F_{(1.50,37.61)}=4.852 p=0.583$ \\
\hline & d' & 3 & Direct & GLM & $F_{(1,25)}=5.526, p=0.027$ & $F_{(2,50)}=2.466, p=0.095$ & $F_{(2,50)}=0.016, p=0.984$ \\
\hline \multirow[t]{2}{*}{ Mental rotation } & Score & 3 & $\begin{array}{l}\text { Transformed (Post1-Pre, } \\
\text { Post2-Pre) }\end{array}$ & ANOVA & - & - & $\begin{array}{l}F_{(1,25)}=0.830, p=0.371 \\
F_{(1,25)}=0.011, p=0.917\end{array}$ \\
\hline & RT & 3 & $\begin{array}{l}\text { Transformed (Post1-Pre, } \\
\text { Post2-Pre) }\end{array}$ & Mann-Whitney & - & - & $\begin{array}{l}U=54.00, p=0.073 \\
U=69.00, p=0.286\end{array}$ \\
\hline \multirow[t]{6}{*}{ Stop-switching } & Go score & 3 & $\begin{array}{l}\text { Transformed (Post1-Pre, } \\
\text { Post2-Pre) }\end{array}$ & $\begin{array}{l}\text { Mann-Whitney (Post1- } \\
\text { Pre) } \\
\text { ANOVA (Post2-Pre) }\end{array}$ & - & - & $\begin{array}{l}U=87.500, p=0.864 \\
F_{(1,25)}=0.014, p=0.907\end{array}$ \\
\hline & Go RT & 3 & Direct & GLM & $F_{(1,25)}=0.175, p=0.679$ & $F_{(2,50)}=3.346, p=0.043$ & $F_{(2,50)}=0.603, p=0.551$ \\
\hline & Stop score & 3 & $\begin{array}{l}\text { Transformed (Post1-Pre, } \\
\text { Post2-Pre) }\end{array}$ & ANOVA & - & - & $\begin{array}{l}F_{(1,25)}=1.134, p=0.297 \\
F_{(1,25)}=1.326, p=0.260\end{array}$ \\
\hline & $\begin{array}{l}\text { Stop signal RT } \\
\text { (SSRT) }\end{array}$ & 3 & $\begin{array}{l}\text { Direct (Pre vs. Post1) } \\
\text { Transformed (Post2-Pre) }\end{array}$ & GLM Mann-Whitney & $\begin{array}{c}F_{(1,25)}=0.089, p=0.767 \\
-\end{array}$ & $F_{(1,25)}=4.298, p=0.049$ & $\begin{array}{l}F_{(1,25)}=0.012, p=0.913 \\
U=75.00, p=0.438\end{array}$ \\
\hline & Switch score & 3 & Direct & GLM & $F_{(1,25)}=0.015, p=0.902$ & $F_{(2,50)}=5.880, p=0.005$ & $F_{(2,50)}=0.023, p=0.977$ \\
\hline & Switch RT & 3 & Direct & GLM & $F_{(1,25)}=1.015, p=0.321$ & $F_{(2,50)}=3.819, p=0.029$ & $F_{(2,50)}=0.213, p=0.809$ \\
\hline \multirow[t]{2}{*}{ Raven } & Score & 2 & Transformed (Post1-Pre) & Mann-Whitney & - & - & $U=81.500, p=0.638$ \\
\hline & $\mathrm{RT}$ & 2 & Direct & GLM & $F_{(1,25)}=7.412, p=0.012$ & $F_{(1,25)}=2.096, p=0.160$ & $F_{(1,25)}=0.592, p=0.449$ \\
\hline \multirow[t]{2}{*}{ Matchstick } & Accuracy & 1 & Direct & $t$-test & $t_{(25)}=0.30, p=0.763$ & - & - \\
\hline & $\begin{array}{l}\mathrm{RT} \text { (correct } \\
\text { answers) }\end{array}$ & 1 & Direct & $t$-test & $t_{(25)}=0.18, p=0.861$ & - & - \\
\hline Five-point & & 1 & Direct & $t$-test & $t_{(25)}=0.94, p=0.358$ & - & - \\
\hline
\end{tabular}

Note: Statistically significant results are marked in bold. GLM, General linear model; RT, Reaction time. 
find out whether these variables could predict the changes in cognitive performance-most especially in executive functions and working memory-after training (i.e., predictors over the difference scores between pre- and post-training assessments).

The model was statistically significant for the 3-back task accuracy $\left(F_{(3,23)}=3.36, p=0.036\right.$, adjusted $\left.\mathrm{R}^{2}=0.21\right)$ and the d' index $\left(F_{(2,23)}=5.23, p=0.007\right.$, adjusted $\left.\mathrm{R}^{2}=0.33\right)$; and the Stop-switching task SSRT $\left(F_{(2,23)}=5.55, p=0.005\right.$, adjusted $\left.\mathrm{R}^{2}=0.34\right)$. In particular, changes observed in the $\mathrm{d}^{\prime}$ index for the 3 -back task were predicted only by early video gaming experience (beta $=0.66, t_{(26)}=3.51, p=0.002$ ), but not by age or gender ( $p$ s $>0.788$ ). Similar results were obtained for the accuracy score for the 3-back task were only the $p$-value was close-to-significant for early video gaming experience (beta $=0.41, t_{(26)}=2.01$, $p=0.056$ ). SSRT changes were influenced by both early video gaming experience (beta $=-0.70, t_{(26)}=-3.78, p=0.001$ ) and gender $\left(\right.$ beta $\left.=-0.57, t_{(26)}=3.15, p=0.004\right)$.

\section{Effects of Video Gaming Experience}

To further explore the influence of early video gaming experience and gender on cognitive performance, we first determined whether there was a gender imbalance between experienced $(n=12)$ and non-experienced $(n=15)$ participants, finding that the difference was statistically significant $\left(\chi_{(1)}^{2}=6.24\right.$; $p=0.013)$, with more male participants having early video gaming experience (69.23\%) than women $(21.43 \%)$.

Then we explored the influence of video gaming experience on the d' score for the 3-back task and the SSRT for the Stop-switching task by dividing the Active and Sham groups into two subgroups each of experienced and non-experienced participants, resulting in the four subgroups described above (see the "Design" and "Demographic Data" sections). For the SSRT we also included gender as a covariate based on the results of the logistic regression.

For the 3-back task, results showed that, when comparing the change in d' scores between pre and postl assessment among groups, there was a statistically significant difference $\left(F_{(3,23)}=5.6, p=0.005, \eta_{p}^{2}=0.42\right)$, where performance in the Active+Exp group was superior to that of Sham+NoExp, despite starting from an equal baseline (see Figure 3). Post hoc analysis revealed differences between the Active+Exp subgroup and the two subgroups without early video gaming experience, namely, Sham $+\operatorname{NoExp}(p=0.048)$ and Active+NoExp $(p=0.012)$. Furthermore, $t$-tests comparing Pre vs. Post1 3-back d' scores within group, revealed significant differences for the two subgroups with early video gaming experience (Sham+Exp: $t_{(5)}=-3.28, p=0.022$ and Active+Exp: $t_{(5)}=-3.36$, $p=0.020)$, but not for the two subgroups without early video gaming experience (Sham+NoExp: $t_{(6)}=-1.32, p=0.236$ and Active+NoExp: $\left.t_{(7)}=1.33, p=0.225\right)$.

For the SSRT, the GLM revealed a main effect of time $\left(F_{(2,44)}=7.71, p=0.001, \eta_{p}^{2}=0.26\right)$, an interaction effect of time by gender $\left(F_{(2,44)}=5.37, p=0.008, \eta_{p}^{2}=0.20\right)$, and a closeto-significant interaction between time and group $\left(F_{(6,44)}=2.2\right.$, $p=0.061, \eta_{p}^{2}=0.23$; see Figure 4). This last interaction effect was due to significant differences between Pre and Post 1 assessments

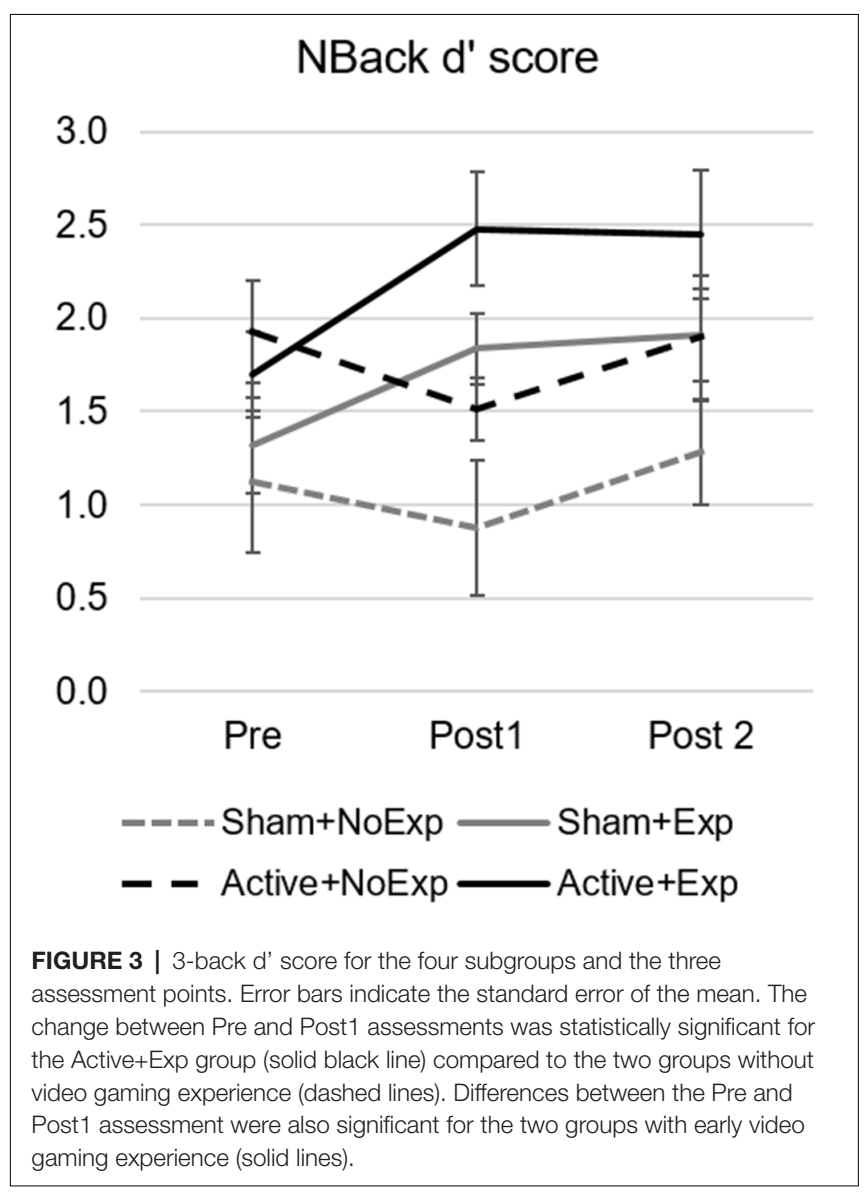

for the two subgroups without video gaming experience $($ Sham + NoExp, $p=0.033$ and Active + NoExp, $p=0.001)$.

\section{Subjective Gaming Experience}

The levels of motivation before and fun and frustration after the training were not significantly different between the Active and Sham groups ( $p s>0.483$ ) or between the four subgroups ( $p$ s $>0.522)$, not even for gender $(p=0.264)$.

\section{DISCUSSION}

This research aimed to assess whether a dual intervention based on video game training and non-invasive brain stimulation could enhance cognitive function in terms of: (1) specific cognitive abilities directly trained during the intervention (i.e., video gaming skills, near-transfer effect); and (2) other cognitive functions not directly trained (i.e., generalization or far-transfer effect). Results showed very limited changes that were mainly related to the near-transfer effect, i.e., variables related to video gaming skills.

The near-transfer effect assessed using an alternative version of the video game that participants played before and after the intervention period, was present in both the Active and Sham stimulation groups. This result combined with the fact that there was no difference between those groups in video game achievements at the end of the training period, seems to 


\section{SSRT + gender (cov)}

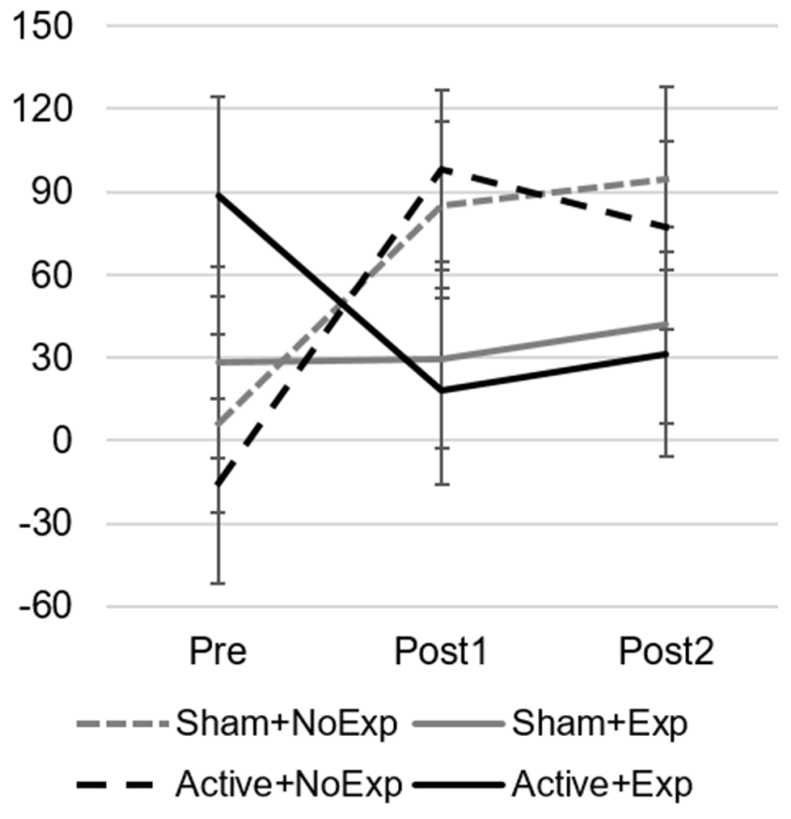

FIGURE 4 | Stop signal reaction times (SSRT) for the Stop-switching task with gender as a covariate for the four subgroups and the three assessment points. Error bars indicate the standard error of the mean. Differences were significant between the Pre and Post1 assessments in the two groups without early video gaming experience (dashed lines).

indicate that the near-transfer effect was only due to the video game training and not mediated by TMS. To our knowledge, there is no literature in which TMS has shown improvements in video gaming performance, although some recent studies have documented positive near-transfer results using transcranial direct current stimulation (e.g., Hsu et al., 2015; Looi et al., 2016). Moreover, the literature showing cognitive improvement in healthy participants using TMS is limited and some studies have shown negative results or subtle effects (e.g., GaudeauBosma et al., 2013; Viejo-Sobera et al., 2017). Even more, the number of studies showing a lack of effect of TMS on cognition may be hard to determine due to strong publication bias.

Regarding the effect of the training period on the evaluated cognitive domains (far-transfer), we observed an improvement in certain cognitive control tasks: digits forward, 3-back task, and accuracy and reaction times for switching trials in the stop-switching task. In contrast, we observed a deterioration in reaction times in go trials and the SSRT of this task. All these effects were present in both the Active and Sham groups, probably due to the video game training and the practice effects present in the neuropsychological tasks, observing, again, the lack of effect of the TMS stimulation. In any case, the near-transfer effects seemed more meaningful since the observed effect size (partial eta squared) in this case was much bigger than that observed in the possible far-transfer effects ( 0.80 vs. $0.12-0.23$, respectively), and the Bayesian analyses only showed weak to moderate evidence to support the alternative hypothesis of far-transfer effect.
Since we did not find an effect of TMS on cognitive performance, we explored other variables that could influence the results. This analysis led us to observe that early video gaming experience may have mediated the improvement in certain cognitive functions. In particular, participants with early video gaming experience improved working memory performance (as evidenced by the increase in the d' index of the 3-back task). This was a surprising result since far-transfer effects on visual working memory tasks as a result of video gaming play have not been widely documented in the literature. We have found one study in which working memory performance was enhanced after video game training in a first-person shooter action game (compared to a simulation-strategy game; Blacker et al., 2014), but another one, which trained in a real-time strategy game failed to find such an effect (Basak et al., 2011). Regarding inhibitory control, we observed that participants without early video gaming experience increased their reaction times while accuracy was maintained in the SSRT of the Stop-Switching task. This is most likely due to a change of strategy to avoid the interference between go, stop, and switch trials, making participants more cautious towards their responses. Experienced players, however, did not show this change in response strategy, maintaining lower reaction times after the training period. This lack of improvement and maintenance of inhibitory ability in experienced gamers has been shown in previous studies (Whitlock et al., 2012; Colzato et al., 2013; Steenbergen et al., 2015).

The similar near- and far-transfer effects found in both stimulation groups, together with the effects observed only in participants with early video gaming experience, corroborate the observation that playing video games may have an impact on cognitive functions in the long term (41-43). The 10-day training period would have made it easier for experienced players to improve their performance in specific executive function domains. In any case, given the small sample size of the subgroups included in the comparison of experienced vs. not experienced gamers, these results should be interpreted as exploratory.

The observed lack of effect of TMS in any of the studied domains has several possible explanations. In the following paragraphs, we will explore different arguments and considerations previously documented in the literature to understand our results.

First, we must consider whether the stimulation protocol (iTBS after training) and the targeted brain region (the DLPFC) were the most appropriate for the specific goals of this research. Although iTBS protocol is still not widely used in research focused on cognitive enhancement, it was chosen for its ability to induce longer-lasting effects over the cerebral cortex with shorter stimulation times compared to rTMS (Goldsworthy et al., 2012; Suppa et al., 2016). A recent systematic review and meta-analysis that assessed the reliability and effectiveness of theta-burst stimulation protocols applied to the prefrontal cortex in healthy participants (Lowe et al., 2018), has shown the variability in the effects. iTBS can somehow modulate executive control, but its effectiveness seems to be task-dependent, being greater for working memory paradigms. Nevertheless, only eight studies have used this paradigm to improve executive functions, so the 
results should be interpreted with caution. Regarding the time of stimulation, we decided to apply it after the training session to improve the effects of the training as shown in animal models (Redolar-Ripoll et al., 2002). Nevertheless, stimulation before performing a specific task is also a common practice in human studies and this shift in time could have affected the results.

We should also consider that the effect of TMS in healthy samples is potentially explained by inter- and intra-individual variables (Hinder et al., 2014; López-Alonso et al., 2014; Suppa et al., 2016; Jannati et al., 2017). These variables include genetic factors (e.g., Cheeran et al., 2008; Li Voti et al., 2011; Mori et al., 2011; Lee et al., 2014), cortical networks organization (Nettekoven et al., 2014, 2015) and age (Müller-Dahlhaus et al., 2008), among other factors. Moreover, the number of sessions can also be a limiting factor in achieving the desired results. Although effects have been observed after 10 sessions of stimulation, interventions in clinical populations usually require more sessions to observe more consistent long-term effects. For example, the mean number of sessions for treating depression is 17 , with a great degree of variability among studies, typically ranging from 9 to 25 sessions (Berlim et al., 2017).

Regarding the target for the stimulation, the right DLPFC, is a relatively large area; nonetheless, the figure-of-eight coil and the navigated stimulation increase the focus and precision of the target location. Given the extensive connectivity of the DLPFC (Sepulcre et al., 2012) and the involvement of different regions of this area in the measured functions, possibly the highly specific target was not directly responsible for the neural processes underlying the cognitive abilities assessed by our tasks. We selected our target based on structural changes observed after playing this particular video game (Kühn et al., 2014) and its involvement in executive functions and working memory, but perhaps our stimulation protocol and/or target did not affect the functional circuits involved in cognitive changes after video game practice (Strenziok et al., 2014; Richlan et al., 2018). This would have been particularly relevant since TMS might be more likely to induce functional than structural changes, especially after a short intervention period.

Brain state-dependency merits special interest, since "any induced neural activity occurs in the context of a baseline neural activity" (Silvanto and Pascual-Leone, 2008). It has been shown that the effects of TMS could be qualitatively modulated by the manipulation of the brain state before the stimulation (Silvanto et al., 2017; e.g., Silvanto and Cattaneo, 2017). In our study, we administered stimulation together with cognitive training to take advantage of the state-dependency phenomenon, and all participants received stimulation immediately after finishing the video gaming period. However, we did not implement any specific control of the ongoing mental state of participants immediately before or during stimulation. Thus, the neural activity could certainly have differed in the participants, leading to uncontrolled or null effects of the stimulation.

Moreover, and considering our results, we must also take into account that the brain network organization of experienced vs. non-experienced gamers has probably led to different brain states while gaming and, thus, when receiving the stimulation after gaming. This might have constituted an advantage for the experienced gamers in terms of responding positively to the stimulation, explaining why the experienced participants who received active TMS showed a greater improvement in the 3-back task compared to both experienced and non-experienced sham groups, and with the active TMS non-experienced group.

Lastly, we need to reflect on the ceiling effect of cognitive enhancement in healthy subjects. In neuropsychology, patients with neurological diseases or psychiatric patients usually have some room for improvement, but in healthy individuals who already perform at, or close to, their full potential, it is difficult to achieve cognitive enhancement. The effects of TMS are linked to the baseline performance of participants, with lower baseline scores associated with higher cognitive facilitation (Silvanto et al., 2018). In the same meta-analysis mentioned above (Lowe et al., 2018), the authors point out that the largest effect size was observed for an older adult population, supporting the idea that iTBS may be more effective in addressing cognitive decline or impairment in clinical or vulnerable populations than in enhancing cognition in healthy ones. Along the same lines, Looi et al. (2016) reported better results for subjects who performed worse at baseline. In our study, participants were healthy, young, and with high education levels, which would leave little room for cognitive improvement. Overall, these and previous results underscore the importance of reconsidering whether efforts in non-invasive brain stimulation research should be aimed at enhancing cognitive performance in healthy individuals, and rise some ethical concerns given the possibly greater potential of these techniques for clinical populations. Nevertheless, we should not forget that the ultimate goal of this study, and many others involving healthy participants, is to apply the knowledge gained in these samples to clinical ones, rather than trying to benefit healthy individuals in no need for these technologies. In any case, the ethical debate must be always present in neuroscience research and especially in the non-invasive stimulation field.

\section{CONCLUSIONS}

This study aimed to test the synergistic effect of the combination of non-invasive brain stimulation and video game training in cognitive enhancement. However, our results did not support this hypothesis, showing an absence of combined effects on cognitive performance. Due to this observed lack of effect, we explored other variables that could have influenced our results showing that early video gaming experience had an impact on the improvement of certain cognitive functions. This result supports the idea that video gaming may modulate cognitive functions in the long term.

Contrary to most studies in the literature where iTBS led to improvements in cognitive tasks at the end of the stimulation, we did not assess cognitive performance immediately after stimulation, as our goal was to determine the presence of transfer effects of the training period. In this scenario, the application of iTBS to the right DLPFC seems to be ineffective in achieving long-term cognitive improvements in healthy patients. Possible explanations may be the poor reliability and effectiveness of the iTBS protocol, highly localized stimulation in a large and widely connected brain area, inter- and intra-individual variability, 
brain state dependency phenomenon, the ceiling effect of cognitive enhancement in healthy subjects, or a combination of some or all of these factors.

Despite not achieving the desired effects of the stimulation, our results, although exploratory, provide valuable information regarding the limitations of stimulating healthy brains and the possible beneficial effects of exposure to video games.

\section{DATA AVAILABILITY STATEMENT}

The dataset generated and analyzed during the current study is available in the institutional repository of the Universitat Oberta de Catalunya (O2), public URL: http://hdl.handle.net/10609/100246 (Palaus et al., 2019).

\section{ETHICS STATEMENT}

The studies involving human participants were reviewed and approved by Universitat Oberta de Catalunya (UOC) Ethics Committee. The patients/participants provided their written informed consent to participate in this study.

\section{AUTHOR CONTRIBUTIONS}

MP, DR-R, and EM conceptualized and designed the study. MP designed the experimental tasks. MP and RV-S acquired

\section{REFERENCES}

Anguera, J. A., Boccanfuso, J., Rintoul, J. L., Al-Hashimi, O., Faraji, F., Janowich, J., et al. (2013). Video game training enhances cognitive control in older adults. Nature 501, 97-101. doi: 10.1038/nature12486

Basak, C., Voss, M. W., Erickson, K. I., Boot, W. R., and Kramer, A. F. (2011). Regional differences in brain volume predict the acquisition of skill in a complex real-time strategy videogame. Brain Cogn. 76, 407-414. doi: 10.1016/j. bandc.2011.03.017

Beck, A. T., Steer, R. A., and Brown, G. K. (1996). Manual for the Beck depression inventory-II. San Antonio, TX: Psychological Corporation.

Bentwich, J., Dobronevsky, E., Aichenbaum, S., Shorer, R., Peretz, R., Khaigrekht, M., et al. (2011). Beneficial effect of repetitive transcranial magnetic stimulation combined with cognitive training for the treatment of Alzheimer's disease: a proof of concept study. J. Neural Transm. 118, 463-471. doi: 10.1007/s00702-010-0578-1

Berlim, M. T., McGirr, A., Rodrigues dos Santos, N., Tremblay, S., and Martins, R. (2017). Efficacy of theta burst stimulation (TBS) for major depression: an exploratory meta-analysis of randomized and sham-controlled trials. J. Psychiatr. Res. 90, 102-109. doi: 10.1016/j.jpsychires.2017. 02.015

Biel, A. L., and Friedrich, E. V. C. (2018). Why you should report bayes factors in your transcranial brain stimulation studies. Front. Psychol. 9:1125. doi: 10.3389/fpsyg.2018.01125

Blacker, K. J., Curby, K. M., Klobusicky, E., and Chein, J. M. (2014). Effects of action video game training on visual working memory. J. Exp. Psychol. Hum. Percept. Perform. 40, 1992-2004. doi: 10.1037/a0037556

Blumberger, D. M., Vila-Rodriguez, F., Thorpe, K. E., Feffer, K., Noda, Y., Giacobbe, P., et al. (2018). Effectiveness of theta burst versus high-frequency repetitive transcranial magnetic stimulation in patients with depression (THREE-D): a randomised non-inferiority trial. Lancet 391, 1683-1692. doi: 10.1016/S0140-6736(18)30295-2

Chan, A.-W., Tetzlaff, J. M., Altman, D. G., Laupacis, A., Gøtzsche, P. C., KrležaJerić, K., et al. (2013). SPIRIT 2013 statement: defining standard protocol items and analyzed the data, and all authors interpreted the results and drafted and reviewed the manuscript. All authors have approved the submitted version and have agreed to both to be personally accountable for the author's contributions and to ensure that questions related to the accuracy or integrity of any part of the work, even ones in which the author was not personally involved, are appropriately investigated, resolved, and the resolution documented in the literature.

\section{FUNDING}

MP received specific funding from the Doctoral School of the Universitat Oberta de Catalunya (UOC), under the IN3-UOC Doctoral Theses Grants Programme 2013-2016 (http://in3.uoc.edu). This work was also supported by Grant PSI2016 80056-P from the Ministerio de Economía y Competitividad (MINECO) of Spain co-funded by European Regional Development Plan: A way to make Europe. The funders had no role in study design, data collection and analysis, decision to publish, or preparation of the manuscript.

\section{SUPPLEMENTARY MATERIAL}

The Supplementary Material for this article can be found online at: https://www.frontiersin.org/articles/10.3389/fnhum. 2020.00235/full\#supplementary-material.

for clinical trials. Ann. Intern. Med. 158:200. doi: 10.7326/0003-4819-158-3201302050-00583

Cheeran, B., Talelli, P., Mori, F., Koch, G., Suppa, A., Edwards, M., et al. (2008). A common polymorphism in the brain-derived neurotrophic factor gene (BDNF) modulates human cortical plasticity and the response to rTMS. J. Physiol. 586, 5717-5725. doi: 10.1113/jphysiol.2008.159905

Colzato, L. S., van den Wildenberg, W. P. M., Zmigrod, S., and Hommel, B. (2013). Action video gaming and cognitive control: playing first person shooter games is associated with improvement in working memory but not action inhibition. Psychol. Res. 77, 234-239. doi: 10.1007/s00426-012 -0415-2

Dobrowolski, P., Hanusz, K., Sobczyk, B., Skorko, M., and Wiatrow, A. (2015). Cognitive enhancement in video game players: the role of video game genre. Comput. Hum. Behav. 44, 59-63. doi: 10.1016/j.chb.2014.11.051

Duecker, F., de Graaf, T. A., Jacobs, C., and Sack, A. T. (2013). Time- and task-dependent non-neural effects of real and sham TMS. PLoS One 8:e73813. doi: 10.1371/journal.pone.0073813

Gaudeau-Bosma, C., Moulier, V., Allard, A. C., Sidhoumi, D., Bouaziz, N., Braha, S., et al. (2013). Effect of two weeks of rTMS on brain activity in healthy subjects during an n-back task: a randomized double blind study. Brain Stimul. 6, 569-575. doi: 10.1016/j.brs.2012.10.009

Goldsworthy, M. R., Pitcher, J. B., and Ridding, M. C. (2012). The application of spaced theta burst protocols induces long-lasting neuroplastic changes in the human motor cortex. Eur. J. Neurosci. 35, 125-134. doi: 10.1111/j.1460-9568. 2011.07924.x

Haatveit, B. C., Sundet, K., Hugdahl, K., Ueland, T., Melle, I., and Andreassen, O. A. (2010). The validity of d prime as a working memory index: results from the bergen n-back task. J. Clin. Exp. Neuropsychol. 32, 871-880. doi: 10.1080/13803391003596421

Hartanto, A., Toh, W. X., and Yang, H. (2016). Age matters: the effect of onset age of video game play on task-switching abilities. Attent. Percept. Psychophys. 78, 1125-1136. doi: 10.3758/s13414-016-1068-9

Hinder, M. R., Goss, E. L., Fujiyama, H., Canty, A. J., Garry, M. I., Rodger, J., et al. (2014). Inter- and intra-individual variability following intermittent theta 
burst stimulation: implications for rehabilitation and recovery. Brain Stimul. 7, 365-371. doi: 10.1016/j.brs.2014.01.004

Hopfner, S., Cazzoli, D., Müri, R. M., Nef, T., Mosimann, U. P., Bohlhalter, S., et al. (2015). Enhancing treatment effects by combining continuous theta burst stimulation with smooth pursuit training. Neuropsychologia 74, 145-151. doi: 10.1016/j.neuropsychologia.2014.10.018

Hsu, W.-Y., Zanto, T. P., Anguera, J. A., Lin, Y.-Y., and Gazzaley, A. (2015). Delayed enhancement of multitasking performance: effects of anodal transcranial direct current stimulation on the prefrontal cortex. Cortex 69, 175-185. doi: 10.1016/j.cortex.2015.05.014

Huang, Y.-Z., Edwards, M. J., Rounis, E., Bhatia, K. P., and Rothwell, J. C. (2005). Theta burst stimulation of the human motor cortex. Neuron 45, 201-206. doi: 10.1016/j.neuron.2004.12.033

Huang, Y.-Z., Rothwell, J. C., Chen, R.-S., Lu, C., and Chuang, W.-L. (2011). The theoretical model of theta burst form of repetitive transcranial magnetic stimulation. Clin. Neurophysiol. 122, 1011-1018. doi: 10.1016/j.clinph.2010. 08.016

Jannati, A., Block, G., Oberman, L. M., Rotenberg, A., and PascualLeone, A. (2017). Interindividual variability in response to continuous thetaburst stimulation in healthy adults. Clin. Neurophysiol. 128, 2268-2278. doi: 10.1016/j.clinph.2017.08.023

JASP Team. (2020). JASP. (Version 0.12.2) [Computer software].

Jeffreys, H. (1961). Theory of Probability. 3rd Edn. Barcelona: Oxford University Press.

Johnson, J. S., Kundu, B., Casali, A. G., and Postle, B. R. (2012). Taskdependent changes in cortical excitability and effective connectivity: a combined TMS-EEG study. J. Neurophysiol. 107, 2383-2392. doi: 10.1152/jn. 00707.2011

Johnson, R. C., McClearn, G. E., Yuen, S., Nagoshi, C. T., Ahern, F. M., and Cole, R. E. (1985). Galton's data a century later. Am. Psychol. 40, 875-892. doi: 10.1037/0003-066X.40.8.875

Kaminski, J. A., Korb, F. M., Villringer, A., and Ott, D. V. M. (2011). Transcranial magnetic stimulation intensities in cognitive paradigms. PLoS One 6:e24836. doi: 10.1371 /journal.pone. 0024836

Knoblich, G., Ohlsson, S., Haider, H., and Rhenius, D. (1999). Constraint relaxation and chunk decomposition in insight problem solving. J. Exp. Psychol. Learn. Mem. Cogn. 25, 1534-1555. doi: 10.1037/0278-7393. 25.6.1534

Koch, G., and Rothwell, J. C. (2009). TMS investigations into the task-dependent functional interplay between human posterior parietal and motor cortex. Behav. Brain Res. 202, 147-152. doi: 10.1016/j.bbr.2009.03.023

Kühn, S., Gleich, T., Lorenz, R. C., Lindenberger, U., and Gallinat, J. (2013). Playing Super Mario induces structural brain plasticity: gray matter changes resulting from training with a commercial video game. Mol. Psychiatry 19, 265-271. doi: 10.1038/mp.2013.120

Kühn, S., Lorenz, R. C., Banaschewski, T., Barker, G. J., Büchel, C., Conrod, P. J., et al. (2014). Positive association of video game playing with left frontal cortical thickness in adolescents. PLoS One 9:e91506. doi: 10.1371/journal. pone. 0091506

Lee, N. J., Ahn, H. J., Jung, K.-I., Ohn, S. H., Hong, J., Kim, Y. J., et al. (2014). Reduction of continuous theta burst stimulation-induced motor plasticity in healthy elderly with COMT Val158Met polymorphism. Ann. Rehabil. Med. 38, 658-664. doi: 10.5535/arm.2014.38.5.658

Lee, J., Oh, E., Sohn, E. H., and Lee, A. Y. (2017). Repetitive transcranial magnetic stimulation treatment in Alzheimer's disease. Brain Stimul. 10:437. doi: 10.1016/j.brs.2017.01.302

Li Voti, P., Conte, A., Suppa, A., Iezzi, E., Bologna, M., Aniello, M. S., et al. (2011). Correlation between cortical plasticity, motor learning and BDNF genotype in healthy subjects. Exp. Brain Res. 212, 91-99. doi: 10.1007/s00221-011-2700-5

Lobo, A., Saz, P., and Marcos, G. (2002). MMSE: Examen Cognoscitivo MiniMental. Madrid: TEA Ediciones.

Looi, C. Y., Duta, M., Brem, A.-K., Huber, S., Nuerk, H.-C., and Cohen Kadosh, R. (2016). Combining brain stimulation and video game to promote long-term transfer of learning and cognitive enhancement. Sci. Rep. 6:22003. doi: $10.1038 /$ srep 22003

López-Alonso, V., Cheeran, B., Río-Rodríguez, D., and Fernández-Del-Olmo, M. (2014). Inter-individual variability in response to non-invasive brain stimulation paradigms. Brain Stimul. 7, 372-380. doi: 10.1016/j.brs.2014.02.004
Lowe, C. J., Manocchio, F., Safati, A. B., and Hall, P. A. (2018). The effects of theta burst stimulation (TBS) targeting the prefrontal cortex on executive functioning: a systematic review and meta-analysis. Neuropsychologia 111, 344-359. doi: 10.1016/j.neuropsychologia.2018.02.004

Luber, B., and Lisanby, S. H. (2014). Enhancement of human cognitive performance using transcranial magnetic stimulation (TMS). NeuroImage 85 , 961-970. doi: 10.1016/j.neuroimage.2013.06.007

Maraver, M. J., Bajo, M. T., and Gomez-Ariza, C. J. (2016). Training on working memory and inhibitory control in young adults. Front. Hum. Neurosci. 10:588. doi: 10.3389 /fnhum.2016.00588

Matsugi, A., Mori, N., Uehara, S., Kamata, N., Oku, K., Mukai, K., et al. (2014). Task dependency of the long-latency facilitatory effect on the soleus H-reflex by cerebellar transcranial magnetic stimulation. Neuroreport 25, 1375-1380. doi: 10.1097/WNR.0000000000000275

Mori, F., Ribolsi, M., Kusayanagi, H., Siracusano, A., Mantovani, V., Marasco, E., et al. (2011). Genetic variants of the NMDA receptor influence cortical excitability and plasticity in humans. J. Neurophysiol. 106, 1637-1643. doi: $10.1152 /$ jn. 00318.2011

Müller-Dahlhaus, J. F. M., Orekhov, Y., Liu, Y., and Ziemann, U. (2008). Interindividual variability and age-dependency of motor cortical plasticity induced by paired associative stimulation. Exp. Brain Res. 187, 467-475. doi: 10.1007/s00221-008-1319-7

Nettekoven, C., Volz, L. J., Kutscha, M., Pool, E.-M., Rehme, A. K., Eickhoff, S. B., et al. (2014). Dose-dependent effects of theta burst rTMS on cortical excitability and resting-state connectivity of the human motor system. J. Neurosci. 34, 6849-6859. doi: 10.1523/JNEUROSCI.4993-13.2014

Nettekoven, C., Volz, L. J., Leimbach, M., Pool, E.-M., Rehme, A. K., Eickhoff, S. B., et al. (2015). Inter-individual variability in cortical excitability and motor network connectivity following multiple blocks of rTMS. NeuroImage 118, 209-218. doi: 10.1016/j.neuroimage.2015.06.004

Nguyen, J.-P., Suarez, A., Kemoun, G., Meignier, M., Le Saout, E., Damier, P., et al. (2017). Repetitive transcranial magnetic stimulation combined with cognitive training for the treatment of Alzheimer's disease. Neurophysiol. Clin. 47, 47-53. doi: 10.1016/j.neucli.2017.01.001

Obeso, I., Robles, N., Marrón, E. M., and Redolar-Ripoll, D. (2013). Dissociating the role of the pre-SMA in response inhibition and switching: a combined online and offline TMS approach. Front. Hum. Neurosci. 7:150. doi: 10.3389/fnhum.2013.00150

Palaus, M. (2018). Cognitive Enhancement by Means of TMS and Video Game Training: Synergistic Effects. Spain: Universitat Oberta de Catalunya

Palaus, M., Marrón, E. M., Viejo-Sobera, R., and Redolar-Ripoll, D. (2017). Neural basis of video gaming: a systematic review. Front. Hum. Neurosci. 11:248. doi: 10.3389/fnhum.2017.00248

Palaus, M., Viejo-Sobera, R., Redolar-Ripoll, D., and Marron, E. M. (2019). Cognitive Enhancement via Neuromodulation and Video Games: Synergistic Effects? [dataset]. UOC Institutional Repository O2.

Philip, N. S., Barredo, J., Aiken, E., Larson, V., Jones, R. N., Shea, M. T., et al. (2019). Theta-burst transcranial magnetic stimulation for posttraumatic stress disorder. Am. J. Psychiatry 176, 939-948. doi: 10.1176/appi.ajp.2019.181 01160

Phillips, A., Jannati, A., Hinchamn, C., Stern, A., and Fried, P. (2019). Neuromodulation by iTBS and 10Hz rTMS compared in healthy and depressed adults. Brain Stimul. 12:520. doi: 10.1016/j.brs.2018.12.709

Rabey, J. M., and Dobronevsky, E. (2016). Repetitive transcranial magnetic stimulation (rTMS) combined with cognitive training is a safe and effective modality for the treatment of Alzheimer's disease: clinical experience. J. Neural Transm. 123, 1449-1455. doi: 10.1007/s00702-016-1606-6

Raven, J. C. (1936). Mental Tests Used in Genetic Studies: The Performance of Related Individuals on Tests Mainly Educative and Mainly Reproductive. London: University of London.

Raven, J. C., and Court, H. J. (2014). Raven, Matrices Progresivas. Madrid: Pearson Clinical.

Redolar-Ripoll, D., Aldavert-Vera, L., Soriano-Mas, C., Segura-Torres, P., and Morgado-Bernal, I. (2002). Intracranial self-stimulation facilitates memory consolidation, but not retrieval: its effects are more effective than increased training. Behav. Brain Res. 129, 65-75. doi: 10.1016/s0166-4328(01)00325-4

Redolar-Ripoll, D., Viejo-Sobera, R., Palaus, M., Valero-Cabré, A., and Marrón, E. M. (2015). Local pain during transcranial magnetic 
stimulation induced by ferromagnetic pigments in commonly used cosmetics. Clin. Neurophysiol. 126, 2243-2245. doi: 10.1016/j.clinph.2015. 03.003

Richlan, F., Schubert, J., Mayer, R., Hutzler, F., and Kronbichler, M. (2018). Action video gaming and the brain: fMRI effects without behavioral effects in visual and verbal cognitive tasks. Brain Behav. 8:e00877. doi: 10.1002/ brb3.877

Romei, V., Thut, G., and Silvanto, J. (2016). Information-based approaches of noninvasive transcranial brain stimulation. Trends Neurosci. 39, 782-795. doi: 10.1016/j.tins.2016.09.001

Rossi, S., Hallett, M., Rossini, P. M., and Pascual-Leone, A. (2009). Safety, ethical considerations and application guidelines for the use of transcranial magnetic stimulation in clinical practice and research. Clin. Neurophysiol. 120, 2008-2039. doi: 10.1016/j.clinph.2009.08.016

Salat, D. H., Kaye, J. A., and Janowsky, J. S. (2002). Greater orbital prefrontal volume selectively predicts worse working memory performance in older adults. Cereb. Cortex 12, 494-505. doi: 10.1093/cercor/12.5.494

Sandrini, M., Umiltà, C., and Rusconi, E. (2011). The use of transcranial magnetic stimulation in cognitive neuroscience: a new synthesis of methodological issues. Neurosci. Biobehav. Rev. 35, 516-536. doi: 10.1016/j.neubiorev.2010. 06.005

Schilberg, L., Brem, A.-K., Freitas, C., Atkinson, N., Asboth, L., Carbone, C., et al. (2012). Effects of cognitive training combined with transcranial magnetic stimulation on cognitive functions and brain plasticity in patients with Alzheimer's disease. Alzheimers Dement. 8:P709. doi: 10.1016/j.jalz.2012. 05.1916

Sepulcre, J., Sabuncu, M. R., Yeo, T. B., Liu, H., and Johnson, K. A. (2012). Stepwise connectivity of the modal cortex reveals the multimodal organization of the human brain. J. Neurosci. 32, 10649-10661. doi: 10.1523/JNEUROSCI.0759 $-12.2012$

Shepard, R. N., and Metzler, J. (1971). Mental rotation of three-dimensional objects. Science 171, 701-703. doi: 10.1126/science.171.3972.701

Silvanto, J., and Cattaneo, Z. (2017). Common framework for "virtual lesion" and state-dependent TMS: the facilitatory/suppressive range model of online TMS effects on behavior. Brain Cogn. 119, 32-38. doi: 10.1016/j.bandc.2017. 09.007

Silvanto, J., and Pascual-Leone, A. (2008). State-dependency of transcranial magnetic stimulation. Brain Topogr. 21, 1-10. doi: 10.1007/s10548-0080067-0

Silvanto, J., Bona, S., and Cattaneo, Z. (2017). Initial activation state, stimulation intensity and timing of stimulation interact in producing behavioral effects of TMS. Neuroscience 363, 134-141. doi: 10.1016/j.neuroscience.2017. 09.002

Silvanto, J., Bona, S., Marelli, M., and Cattaneo, Z. (2018). On the mechanisms of Transcranial Magnetic Stimulation (TMS): how brain state and baseline performance level determine behavioral effects of TMS. Front. Psychol. 9:741. doi: $10.3389 /$ fpsyg.2018.00741

Steenbergen, L., Sellaro, R., Stock, A.-K., Beste, C., and Colzato, L. S. (2015). Action video gaming and cognitive control: playing first person shooter games is associated with improved action cascading but not inhibition. PLoS One 10:e0144364. doi: 10.1371/journal.pone.0144364

Strenziok, M., Parasuraman, R., Clarke, E., Cisler, D. S., Thompson, J. C., and Greenwood, P. M. (2014). Neurocognitive enhancement in older adults: comparison of three cognitive training tasks to test a hypothesis of training transfer in brain connectivity. NeuroImage 85, 1027-1039. doi: 10.1016/j. neuroimage.2013.07.069

Suppa, A., Huang, Y.-Z., Funke, K., Ridding, M. C., Cheeran, B., Di Lazzaro, V., et al. (2016). Ten years of theta burst stimulation in humans: established knowledge, unknowns and prospects. Brain Stimul. 9, 323-335. doi: 10.1016/j. brs.2016.01.006

Taatgen, N. A. (2013). The nature and transfer of cognitive skills. Psychol. Rev. 120, 439-471. doi: 10.1037/a0033138

Tucha, L., Aschenbrenner, S., Koerts, J., and Lange, K. W. (2012). The five-point test: reliability, validity and normative data for children and adults. PLoS One 7:e46080. doi: 10.1371/journal.pone.0046080

Viejo-Sobera, R., Redolar-Ripoll, D., Boixadós, M., Palaus, M., Valero-Cabré, A., and Marrón, E. M. (2017). Impact of prefrontal theta burst stimulation on clinical neuropsychological tasks. Front. Neurosci. 11:462. doi: 10.3389/fnins. 2017.00462

Wechsler, D. (2008). Wechsler Adult Intelligence Scale-Fourth Edition. San Antonio, TX: The Guilford Press.

Whitlock, L. A., McLaughlin, A. C., and Allaire, J. C. (2012). Individual differences in response to cognitive training: using a multi-modal, attentionally demanding game-based intervention for older adults. Comput. Hum. Behav. 28, 1091-1096. doi: 10.1016/j.chb.2012.01.012

Zhao, X., Wang, H., and Maes, J. H. R. (2020). Training and transfer effects of extensive task-switching training in students. Psychol. Res. 84, 389-403. doi: $10.1007 / \mathrm{s} 00426-018-1059-7$

Conflict of Interest: The authors declare that the research was conducted in the absence of any commercial or financial relationships that could be construed as a potential conflict of interest.

Copyright (๑) 2020 Palaus, Viejo-Sobera, Redolar-Ripoll and Marrón. This is an open-access article distributed under the terms of the Creative Commons Attribution License (CC BY). The use, distribution or reproduction in other forums is permitted, provided the original author(s) and the copyright owner $(s)$ are credited and that the original publication in this journal is cited, in accordance with accepted academic practice. No use, distribution or reproduction is permitted which does not comply with these terms. 